\title{
CONCEPTS FOR A SLOW-POSITRON TARGET AT THE ADVANCED PHOTON SOURCE CONF-970503--
}

\author{
E. Lessner and M. White \\ Argonne National Laboratory, 9700 South Cass Avenue, Argonne, Illinois 60439-4800 USA
}

\section{Abstract}

The Advanced Photon Source (APS) [1,2] linear accelerator beam could be used to produce slow positrons during the hours between the storage ring injection cycles. Initial concepts for the design of a target that is optimized for slow-positron production are discussed, and simulation results are presented. Some possible ways to increase the nominal linac beam power for improved slow-positron production are also discussed.

\section{INTRODUCTION}

Slow positrons are valuable tools in atomic physics, materials science, and solid state physics research. Slow positrons can be used: 1) to probe defects in metals, as positrons are repelled by ionized atoms and may be captured at vacancies; 2) to study Fermi surfaces through analysis of photons generated by positron/electron annihilation; and 3 ) to study surfaces and interfaces of materials through analysis of energy losses, diffraction, and reemission of positrons from surfaces or interfaces.

Slow positrons can be emitted by radioisotope sources or they can be obtained by moderating the positrons produced by accelerator beams. Normally, an intense electron beam impinges on a target made of a high atomic number material, such as tungsten or tantalum. Positron production occurs as a result of bremsstrahlung interactions, and the positrons are then moderated by a series of foils that have a negative work-function for positrons. Positrons emitted from the moderator are then captured and transported to an experimental area by electromagnetic fields. The number of positrons that can be delivered to an experiment is a function of the incident beam power, target material and geometry, moderator efficiency, and slow positron capture and transport efficiency.

The APS linac beam could be used to produce slow positrons during the hours between storage ring injection cycles or top-off operations. The linac and some possible ways of increasing its beam power are discussed.

Initial concepts for the design of a target that is optimized for slow-positron production are discussed, and simulation results are presented. We compare the positron yield obtained from simulations of various target configurations for a fixed beam power and energy. Finally, we present an integrated target-moderator concept that will result in a high-intensity slow positron source, when combined with an efficient extraction and transport system.

\section{THE APS LINAC}

The APS electron linac accelerates 30-ns-long pulses containing $50 \mathrm{nC}$ of charge to an energy of $200 \mathrm{MeV}$. The resulting $500-\mathrm{W}$ electron beam impinges on a 7 -mm-thick water-cooled tungsten target that serves as a positron converter. Pair-produced positrons and electrons are refocused by a 1.5-T pulsed coil and directed into the positron linac where, during normal operation, they are captured and accelerated to $450 \mathrm{MeV}$. Linac design parameters are listed in Table 1 , together with achieved performance values.

The nominal electron beam power of $500 \mathrm{~W}$ can be increased for slow positron production purposes by increasing the nominal pulse length of 30 ns and by increasing the effective repetition rate.

The upstream accelerating structure in each linac is directly powered by a $35-\mathrm{MW}$ klystron, while the remaining structures are powered in groups of four by a klystron and SLED (SLAC Energy Doubler) cavity assembly. The SLED cavities can be detuned, thereby allowing the full klystron pulse, nominally $5 \mu \mathrm{s}$, to be used. The beam energy with SLEDs detuned but without heavy beam loading was measured to be about $400 \mathrm{MeV}$. Measurements to determine the maximum accelerated pulse length with reasonable energy spread are scheduled. A new pulser design that allows re-firing of the gun $1 \mu \mathrm{s}$ after the end of the first beam pulse is under consideration.

The linac repetition rate is limited to $60 \mathrm{~Hz}$ by the present modulator's resonant-charging system design; however constant-current power supplies that could also allow a faster rate are presently being tested [3].

Table 1: Linac Performance Summary

\begin{tabular}{lccc}
\hline & Design & Achieved & Units \\
\hline Energy on Target & 200 & 240 & $\mathrm{MeV}$ \\
Gun Pulse Length & $30 \mathrm{~ns}$ & $>1 \mu \mathrm{s}$ & \\
Current on Target & 1.7 & $>2$ & $\mathrm{~A}$ \\
Rf Rate & 60 & 60 & $\mathrm{~Hz}$ \\
$\mathrm{e}^{-}$Energy Spread & \pm 8 & $\leq \pm 8$ & $\%$ \\
$\mathrm{e}^{+}$Emittance & $\leq 1.2$ & $\leq 1.2$ & $\mathrm{~mm} \mathrm{mrad}$ \\
$\mathrm{e}^{+}$Energy & 450 & 458 & $\mathrm{MeV}$ \\
$\mathrm{e}^{+}$Current & 8 & 14 & $\mathrm{~mA}$ \\
$\mathrm{e}^{+}$Energy Spread & \pm 1 & $\leq \pm 1.6$ & $\%$ \\
\hline
\end{tabular}

The submitted manuscript has been created by the University of Chicago as Operator of Argonne National Laboratory ("Argonne") under Contract No. W-31-109-ENG-38 with the U.S. Department of Energy. The L.S. Government retains for itself, and others acting on its behalf, a paid-up, nonexclusive, irrevocable worldwide license in said article to reproduce, prepare derivative works. distribute copies to the public, and perform publicly and display publicly, by or on behalf of the Government. 


\section{DISCLAIMER}

This report was prepared as an account of work sponsored by an agency of the United States Government. Neither the United States Government nor any agency thereof, nor any of their employees, makes any warranty, express or implied, or assumes any legal liability or responsibility for the accuracy, completeness, or usefulness of any information, apparatus, product, or process disclosed, or represents that its use would not infringe privately owned rights. Reference herein to any specific commercial product, process, or service by trade name, trademark, manufacturer, or otherwise does not necessarily constitute or imply its endorsement, recommendation, or favoring by the United States Government or any agency thereof. The views and opinions of authors expressed herein do not necessarily state or reflect those of the United States Government or any agency thereof. 


\section{DISCLAMIER}

Portions of this document may be illegible in electronic image products. Images are produced from the best available original document. 


\section{TARGET DESIGN SIMULATIONS}

We performed computer simulations of several target configurations using the electromagnetic shower code EGS4 [4], in order to optimize the target-moderator design parameters. In the simulations, a pencil beam of electrons is incident perpendicularly to the basis of a tungsten cylinder. The beam power is fixed at $800 \mathrm{~W}$ and the incident electron energy is $400 \mathrm{MeV}$. We examined the positron yield from single-layer targets and multiplelayer targets of varying layer thicknesses. The energy distributions and the divergences of all shower products were analyzed in each case.

Simulations of single-layer target geometries indicate that a tungsten target that is three radiation lengths (Xo) thick ( $10.5 \mathrm{~mm}$ thick) results in the highest positron yield. However, for the same incident beam power and energy, the total positron yield from segmented targets can be significantly higher. The total yield of positrons with energies up to $6 \mathrm{MeV}$ is shown in Figure 1. The yields in the figure are calculated per incident electron and are shown for the optimized single-layer target and for the optimized five-layer target. The single-layer target is three-radiation-lengths thick, and the multi-layer target segments are 1.250-, 0.750-, 0.750-, 0.125-, and 0.125radiation-lengths thick, respectively.

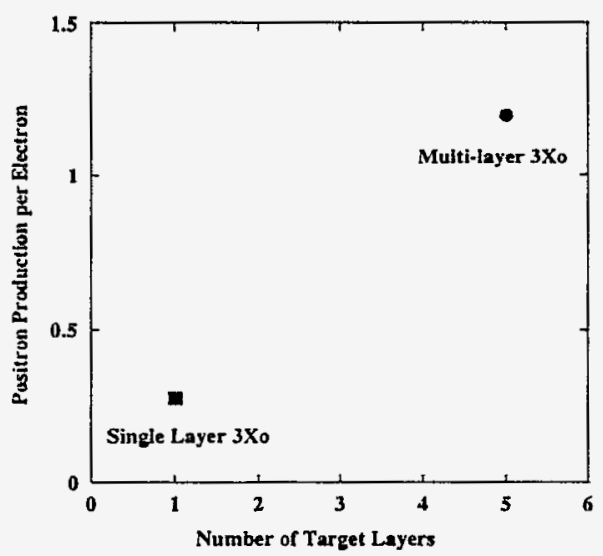

Figure 1: Production, per $400-\mathrm{MeV}$ electron, of positrons with energies in the range $0 \leq \mathrm{E}_{\mathrm{e}}+\leq 6 \mathrm{MeV}$, by a singlesegment three-radiation-length target and by a fivesegment target of the same total effective length.

In Figure 2 we show positron production by each segment of the five-layer target. The yield per incoming $400-\mathrm{MeV}$ electron increases from 0.14 positrons per electron after the first segment to 0.30 after the fourth segment, and decreases slightly to 0.24 after the last segment. Contributions from backscattered positrons are negligible, and are not included in this estimate. Using the numbers above and assuming an average moderator reemission efficiency of $10^{-2}$ [5], we estimate a total slow positron yield from the target of roughly $10^{10}$ positrons per second. The final slow positron current at the experiment will, of course, be significantly less as a result of inefficiencies in the capture and transport processes.

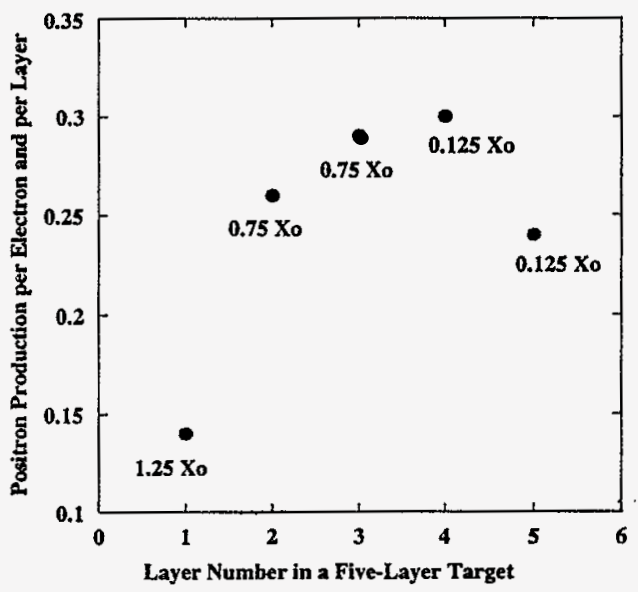

Figure 2: Positron production in the range $0 \leq \mathrm{E}_{\mathrm{e}}+\leq 6 \mathrm{MeV}$ after each target segment of the optimized five-layer target. The segment thicknesses are indicated under each symbol.

The transverse distributions of positrons generated in the first and last segments are shown in Figures 3(a) and 3(b), respectively. The positron beam divergence increases by a factor of ten between the first and the last target segment, as can be seen in the figures.
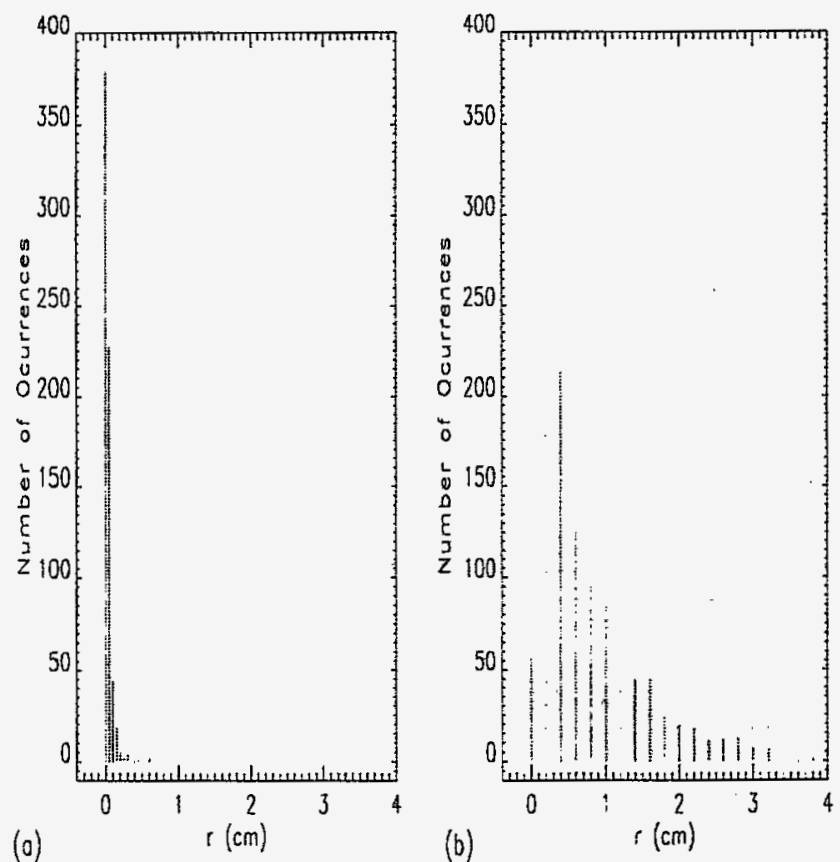

Figure 3: Transverse distributions of positrons with $\left(0 \leq \mathrm{E}_{\mathrm{e}}+\leq 6 \mathrm{MeV}\right)$ generated in the first (a) and fifth (b) segments of the five-layer target. 


\section{A TARGET-MODERATOR CONFIGURATION}

The efficiency of slow positron production depends strongly on the target-moderator geometry. We are developing an integrated tungsten-based target-moderator concept in which the moderator "foils" are machined into the target itself. Figure 4 depicts such a self-moderating tungsten target segment. The reemission percentage of low-energy positrons would be higher if we were to use thinner foils, but thinner foils are difficult to produce and to handle. Our concept uses the electrical discharge machining (EDM) process to machine foils into the target in a simple and straightforward way, thus reducing the manufacturing and handling difficulties. We have shown that foil thicknesses $\geq 125 \mu \mathrm{m}$ can be machined, and we are continuing to investigate ways to achieve thinner foils.

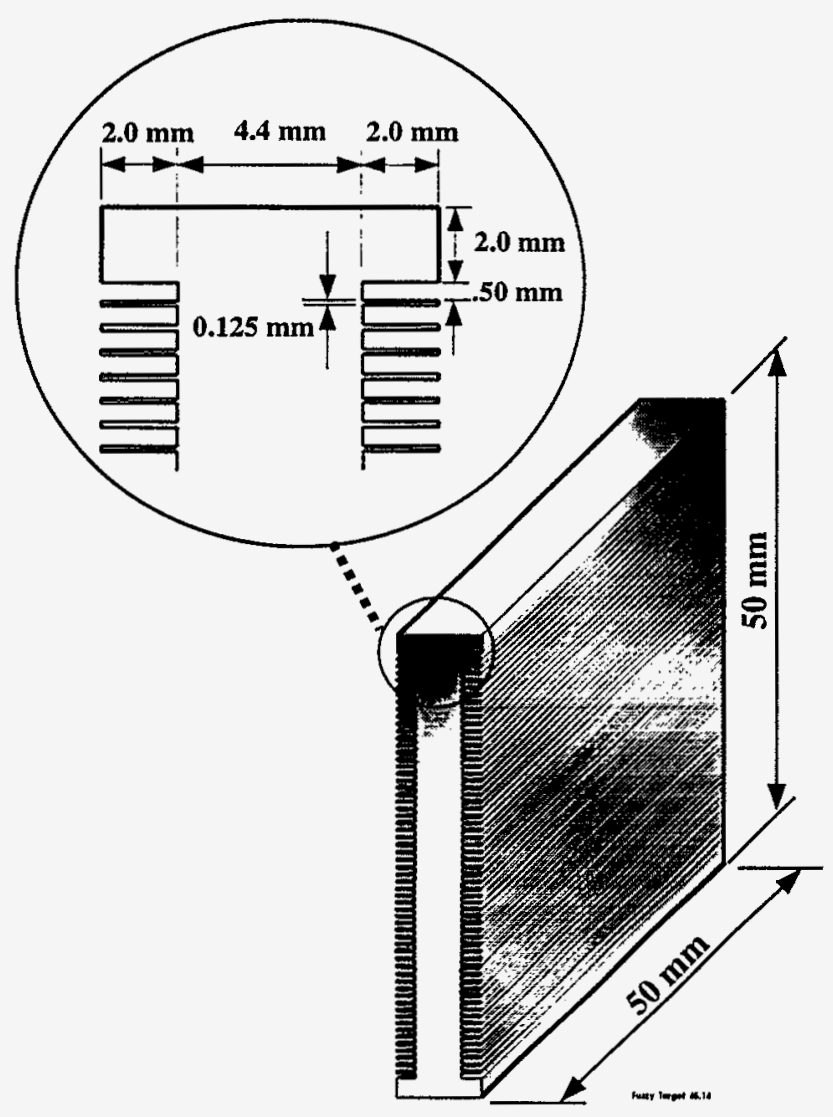

Figure 4: Self-moderating tungsten target segment.

Details of the moderator are shown in the insert.

The separation between foils must be optimized for maximum efficiency while still allowing penetration of the electromagnetic extraction fields. We incorporated effects of the electrical extraction fields into our computer simulation, and preliminary tests of the code have been performed. We have not yet simulated the moderating process.
Our studies indicate that the APS linac can be used as a source of slow positrons. An integrated target-moderator concept is now being optimized, and measurements to determine the maximum achievable linac beam power are underway.

\section{ACKNOWLEDGMENTS}

Work is supported by the U.S. Department of Energy, Office of Basic Energy Sciences, under Contract No. W31-109-ENG-38. We acknowledge L. Emery (ANL) for the use of his C-program interface to EGS4 and P. K. Job for valuable discussions, K. J. Peterson for drawings, D. Mangra for mechanical assistance, and J. N. Galayda and L. C. Teng for continuing support.

\section{REFERENCES}

[1] 7-GeV Advanced Photon Source Conceptual Design Report, ANL-87-15, April 1987.

[2] M. White et al., "Construction, Commissioning, and Operation of the Advanced Photon Source (APS) Linear Accelerator," Proc. of the XVIII International Linac Conference, Geneva Switzerland, 26-30 August 1996, pp. 315-319.

[3] R. Fuja et al., "Constant-Current Charging Supplies for the Advanced Photon Source (APS) Linear Accelerator Modulators," these proceedings.

[4] W. R. Nelson, H. Hirayama, D. W. Rogers, "The EGS4 Code System," SLAC-265 (1985).

[5] S. Okada and H. Sunaga, Nuclear Instruments and Methods, B56/57, pp. 604-609 (1991). 